\title{
The Harrison Diffusion Kinetics Regimes in Grain Boundary Diffusion: Lattice Monte Carlo Modelling of the Effect of Segregation
}

\author{
Irina V. Belova ${ }^{1, a}$, Graeme E. Murch ${ }^{1, b}$ and Thomas Fiedler ${ }^{1, c}$ \\ ${ }^{1}$ Centre for Mass and Thermal Transport in Engineering Materials \\ Priority Research Centre for Geotechnical and Materials Modelling \\ School of Engineering \\ University of Newcastle, Callaghan, NSW 2308, Australia \\ arina.Belova@newcastle.edu.au bGraeme.Murch@newcastle.edu.au \\ cThomas.Fiedler@newcastle.edu.au
}

Keywords: grain boundary diffusion, effective diffusion, Harrison regimes

\begin{abstract}
The location of the transition points for the three main Harrison's kinetics regimes (Type$\mathrm{A}, \mathrm{B}$ and $\mathrm{C}$ ) for the measurement of grain boundary diffusivities from tracer concentration depth profiles (self-diffusion) has been extensively studied in (Divinski et al. Zeit. Metallk, 2002, Belova and Murch, Phil. Mag, 2001, 2009; Defect and Diffusion Forum, 2006, 2008, 2009) by making use of the phenomenological Lattice Monte Carlo numerical method. Those locations are mainly dependent on the dimensionality of the problem. For the case of impurity grain boundary diffusion, the segregation effect is very important. In the present study, the influence of segregation on the transition points is investigated for the parallel slab model (2-dimensional) of the grain boundary diffusion problem by making use of the tracer-type solution to the equivalent diffusion problem. It is shown that the Type-B regime is most likely not realized for the cases of fine-grain material with a strong segregation effect present.
\end{abstract}

\section{Introduction}

According to the Harrison classification [1] there are three principal regimes in the transient grain boundary diffusion problem. The Harrison Type-A kinetics regime (long diffusion times) refers to the situation where the average bulk diffusion length of tracer atoms coming from the tracer source is substantially greater than the grain size $(d)$. The Harrison Type-B kinetics regime (intermediate times) refers to a situation where the average bulk diffusion length of the tracer atoms is less than the grain size. Finally, the Harrison Type-C kinetics regime (very short times) refers to a situation where the bulk diffusion length of the tracer atoms is less than the grain boundary width $\delta$. This classification is valid for tracer diffusion experiments.

The transition points between the Harrison kinetics regimes are of great importance. Numerous previous studies $[2-7,8]$ have shown that these transitions usually occur gradually, not abruptly. Because of this, for the proper analysis of the transient grain boundary diffusion problem additional intermediate, Type-AB [9] and Type-BC [8] kinetics regimes have been introduced. Recent results for the Harrison classification for tracer diffusion and for the parallel slab model can be summarized as follows:

Making use of extensive Lattice Monte Carlo (LMC) calculations of tracer depth concentration profiles as a function of the distance between grain boundaries, the present authors [2] have shown that the transition point from Harrison Type-A to Type-AB kinetics regimes was greatly different from the previously estimated point and occurs when:

$$
\Lambda=\frac{d}{\sqrt{D_{\ell} t}}=0.4,
$$


where $D_{\ell}$ is the lattice (grain) diffusivity and $t$ is the diffusion time. For the transition between Type-AB kinetics and Harrison Type-B kinetics our LMC calculations [3] gave:

$$
\Lambda=3.0
$$

Therefore an intermediate Type-AB regime can be expected to occur between

$$
0.4<\Lambda<3.0
$$

The transition point where the Harrison Type-B kinetics regime last occurs (transition point between the Harrison Type-B kinetics and the Type-BC kinetics) has been estimated to occur at:

$$
\alpha=\frac{\delta}{2 \sqrt{D_{\ell} t}}=0.1
$$

(where $\delta$ is usually assumed to be constant for most materials and is about $0.5 \mathrm{~nm}$.) The transition point where the Harrison Type-C kinetics regime first occurs (transition point between the Type-BC kinetics and the Harrison Type-C kinetics) has been estimated to occur at:

$$
\alpha=5.0 .
$$

Therefore an intermediate Type-BC regime can be expected to occur between:

$$
0.1<\alpha<5.0 \text {. }
$$

In this paper, we consider Harrison kinetics regimes classification for the impurity grain boundary diffusion experiment. We will analyse impurity diffusion where the segregation of the impurity atoms to the grain boundaries is present but we assume that there is no impurity saturation at the grain boundaries. Thus we will take into our considerations only the so-called linear segregation effect. We will consider here only the parallel slab model.

If the impurity diffusion experiment is in the Harrison Type-A kinetics regime, then the corresponding logarithm of the tracer concentration vs $y^{2}$ (where $y$ is the depth in the diffusion

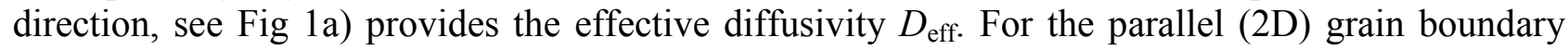
slab diffusion model, $D_{\text {eff }}$ can be analytically represented exactly as a simple weighted arithmetic average (the Modified Hart Equation [10]) of the lattice and the grain boundary diffusivities, $D_{\ell}$ and $D_{\mathrm{gb}}$ respectively with the segregation factor $s$ being taking into account.

If the impurity diffusion experiment is in the Harrison Type-B kinetics regime then the corresponding concentration depth profile usually has two regions. The first region provides the lattice diffusivity whilst the second or tail region provides the so-called triple product of the segregation factor $s$, the grain boundary width and the grain boundary diffusivity, i.e. $s \delta D_{\mathrm{gb}}$.

If the tracer diffusion experiment is in the Harrison Type-C kinetics regime conditions then the corresponding logarithm of the tracer concentration vs. $y^{2}$ provides the grain boundary diffusivity $D_{\mathrm{gb}}$. This can be considered as a short time limit condition where the effective diffusivity can be calculated from the Modified Hart Equation with the segregation factor $s$ going to infinity. It is easy to see that:

$$
D_{e f f}=\frac{\delta s D_{g b}+(d-\delta) D_{\ell}}{\delta s+d-\delta}=D_{g b} .
$$




\section{Model(s)}

First, we consider a model for the impurity grain boundary diffusion problem that can be represented by equally spaced row of parallel slabs of width $\delta$ with the segregation factor $s$ taken into account. Let the $x$ - axis be normal to the grain boundary slab and the $y$-axis be normal to the free surface (Fig 1a). The diffusivity in the grain boundary $D_{\mathrm{gb}}$ is much higher than in the crystal $D_{\ell}: D_{\mathrm{gb}} \gg D_{\ell}$. Then, assuming that Fick's laws of diffusion are valid in both regions and that the diffusion coefficients $D_{\mathrm{gb}}$ and $D_{\ell}$ are isotropic and independent of position and time, we can write the following system of equations (Model I) [11-13]:

$$
\begin{aligned}
& \frac{\partial c_{b}(x, y, t)}{\partial t}=D_{g b}\left(\frac{\partial^{2} c_{b}}{\partial x^{2}}+\frac{\partial^{2} c_{b}}{\partial y^{2}}\right) \text { in the grain boundary }(0 \leq x \leq \delta / 2) ; \\
& \frac{\partial c_{g}(x, y, t)}{\partial t}=D_{\ell}\left(\frac{\partial^{2} c_{g}}{\partial x^{2}}+\frac{\partial^{2} c_{g}}{\partial y^{2}}\right) \text { in the grain }(\delta / 2 \leq x \leq d / 2) .
\end{aligned}
$$

The boundary conditions at $x=0$ and at $x=d / 2$ are simply symmetry conditions (zero flux). The boundary condition at $y=0$ is the condition of instantaneous source consistent with the segregation conditions. At $x=\delta / 2$ we have the following conditions:

$$
\begin{aligned}
& c_{b}(y, t)_{x=\delta / 2}=s c_{g}(y, t)_{x=\delta / 2}, \\
& \left.D_{g b} \frac{\partial c_{b}(x, y, t)}{\partial x}\right|_{x=\delta / 2}=\left.D_{\ell} \frac{\partial c_{g}(x, y, t)}{\partial x}\right|_{x=\delta / 2} .
\end{aligned}
$$

In the present study, we assume that at the (instantaneous) source plane the impurity has been partitioned according to the segregation factor as follows:

$$
c_{b}(y, 0)_{x<\delta / 2}=s c_{g}(y, 0)_{x \delta / 2}=M \delta_{D}(y)
$$

where $\delta_{D}(y)$ is the Dirac delta function defined as $\delta_{D}(y=0)=\infty$; and $\delta_{D}(y \neq 0)=0$.

After some algebraic manipulations and making use of Taylor series expansions for $c_{\mathrm{b}}$ across the grain boundary width (in the $\mathrm{x}$-direction as outlined in [8]) it can be shown (see Appendix) that this problem is equivalent up to second order in $\delta$ to the following (see Fig 1b) Model II:

$$
\begin{aligned}
& \frac{\partial c_{b 1}(x, y, t)}{\partial t}=D_{g b}\left(\frac{\partial^{2} c_{b 1}}{\partial x^{2}}+\frac{\partial^{2} c_{b 1}}{\partial y^{2}}\right) \text { in the grain boundary }(0<x \leq s \delta / 2) ; \\
& \frac{\partial c_{g}(x, y, t)}{\partial t}=D_{\ell}\left(\frac{\partial^{2} c_{g}}{\partial x^{2}}+\frac{\partial^{2} c_{g}}{\partial y^{2}}\right) \text { in the grain }(\mathrm{s} \delta / 2 \leq x \leq(d+(s-1) \delta) / 2) .
\end{aligned}
$$

At $x=s \delta / 2$ we have the following conditions:

$$
\begin{aligned}
& c_{b 1}(y, t)_{x=s \delta / 2}=c_{g}(y, t)_{x=s \delta / 2}, \\
& \left.D_{g b} \frac{\partial c_{b 1}(x, y, t)}{\partial x}\right|_{x=s \delta / 2}=\left.D_{\ell} \frac{\partial c_{g}(x, y, t)}{\partial x}\right|_{x=s \delta / 2} .
\end{aligned}
$$

The conditions at the source plane are: 


$$
c_{b}(y, 0)_{x<\delta / 2}=c_{g}(y, 0)_{x>\delta / 2}=M \delta_{D}(y) .
$$

Accordingly, we can accept that the concentration profiles obtained as solutions to Model I can be very closely approximated by the concentration profiles for the corresponding solutions to Model II. This means that at time $t, D_{\mathrm{gb}}, D_{\ell}$ and the internal grain width $d-\delta$ are the same for both models and the difference is only in the segregation factor s and the grain boundary 'effective' width.

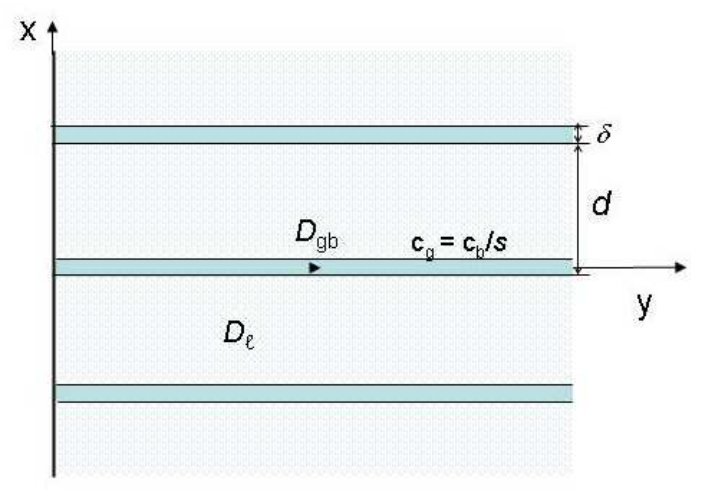

(a)

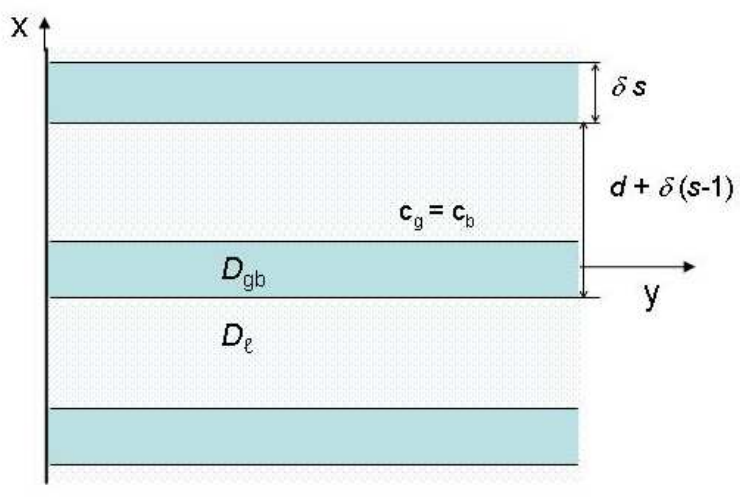

(b)

Figure 1. Schematic presentation of Model I (a) and Model II (b).

This proposition has a very important consequence: It immediately follows that the Harrison classification for Model I should be the same as the Harrison classification for Model II. And this classification (Model II) is equivalent to the tracer case classification summarized above! The only change is that instead of $\Lambda$ and $\alpha$ we need to use ('effective') parameters $\Lambda^{*}$ and $\alpha^{*}$ :

$$
\Lambda^{*}=\frac{L+(s-1) \delta}{\sqrt{D_{\ell} t}} ; \quad \alpha^{*}=\frac{s \delta}{2 \sqrt{D_{\ell} t}} .
$$

It should be noted that the use of an effective $\alpha^{*}$ is already widely accepted and therefore its significance is obvious. But an effective $\Lambda^{*}$ has not been introduced previously. The significance of an effective $\Lambda^{*}$ is that it is now a controlling parameter for the determination of the Type-A, B kinetics regime and the intermediate Type- $\mathrm{AB}$ kinetics regime.

Another few very important points can be made here:

1. First, the boundary condition at the source plane that the impurity atoms are partitioned according to the segregation factor, plays a great role in the equivalence of the solutions of the two Models. If this condition is not valid then, obviously, the time-dependent segregation (or non-equilibrium, 'undeveloped' segregation) will be observed in a given depth profile: less segregation would have to be expected at the beginning of the profile - close to the source plane and more developed segregation would be expected at the tail section given the overall underestimated segregation factor and possibly change in the kinetics transition points. Obviously, the equivalence of Models I and II will be lost because it is based on the assumption that $s$ is constant.

2. Models I and II have identical concentration depth profiles in the y-direction only for the parallel slab geometry. These two models will never provide very similar profiles for closed grain geometries. This can easily be seen if we inspect the (Maxwell [14-15]) expression for the closed grains effective diffusivity in the Type-A kinetics regime:

$$
D_{e f f}^{c l . g r .}=\frac{s g D_{g b}}{g s+1-g}\left(1+\frac{3\left(D_{\ell}-s D_{g b}\right)(1-g)}{2 s D_{g b}+D_{\ell}-\left(D_{\ell}-s D_{g b}\right)(1-g)}\right),
$$


where $1-g$ is the volume fraction of grains in the sample. We can see that (because of the essential non-linearity of the diffusivity terms) it is not possible to obtain the same expression by simply expanding the grain boundaries from width $\delta$ to a width $s \delta$.

\section{Numerical simulations}

Our first computational task is to demonstrate that the concentration profiles calculated as solutions to Models I and II (parallel slab geometry!) are very similar, if not identical, in all the kinetics regimes $(\mathrm{A}, \mathrm{AB}, \mathrm{B}, \mathrm{BC}$ and $\mathrm{C})$. Our second computational task is to show that $\Lambda^{*}$ and $\alpha^{*}$ are the correct parameters for classification purposes. In the Type-A regime the effective diffusivity $D_{\text {eff }}$ as obtained from the Gaussian analysis of the profile, is exactly the same for both models:

$$
\begin{aligned}
& D_{e f f}^{I}=\frac{1}{s \delta+d-\delta}\left(s \delta D_{g b}+(d-\delta) D_{\ell}\right) \\
& D_{e f f}^{I I}=\left(\frac{s \delta}{s g+d-\delta} D_{g b}+\frac{d-\delta}{s g+d-\delta} D_{\ell}\right) \equiv D_{e f f}^{I} .
\end{aligned}
$$

This can be accepted as a test for the equivalence of the models and we can safely assume that in the Type-A regime both profiles then would have to be the same.

Similarly, in the Type- $\mathrm{C}$ regime the Gaussian analysis will give us the grain boundary diffusivity $D_{\mathrm{gb}}$ and this should be true for both models.

In the analysis of the Type- $\mathrm{B}$ regime and the intermediate Types- $\mathrm{AB}$ and $\mathrm{BC}$ regimes there is usually a more complicated situation of the concentration depth profiles. This is why we will attempt to check these regimes more carefully.

\section{Method of Computation}

The present numerical study was performed by making use of the LMC method, see [16]. The grain boundary is represented by regular lattice sites on the lattice planes, for details see [2], in which the grain boundary diffusivity of the diffusing particles is associated with a single jump frequency $\left(w_{\mathrm{gb}}\right)$. The rest of the lattice represents the grains. There, the jump frequency $\left(w_{1}\right)$ is associated with the bulk diffusivity of the diffusing particles. For the segregation effect to be simulated we introduce $\mathrm{w}_{3}-\mathrm{a}$ jump frequency for a particle to jump from the grain boundary plane to a grain site. The ratio of $\mathrm{w}_{1} / \mathrm{w}_{3}$ is then set equal to $\mathrm{s}$. To simulate a thin-film (instantaneous) tracer source, $10^{6}$ $10^{8}$ particles are created and released from the surface (according to the segregation effect for the purposes of avoiding the possible time-dependent segregation) and allowed to diffuse for a time $t$ (proportional to the number of jump attempts per particle).

The principal outputs of the LMC calculations are the tracer concentration (depth) profiles for the grain boundary diffusion which appear in exactly the same fashion as in an experimental tracer diffusion measurement. These concentration profiles are built up simply by determining the number of particles that have reached a given distance from the tracer source plane after the diffusion time $t[16]$. 


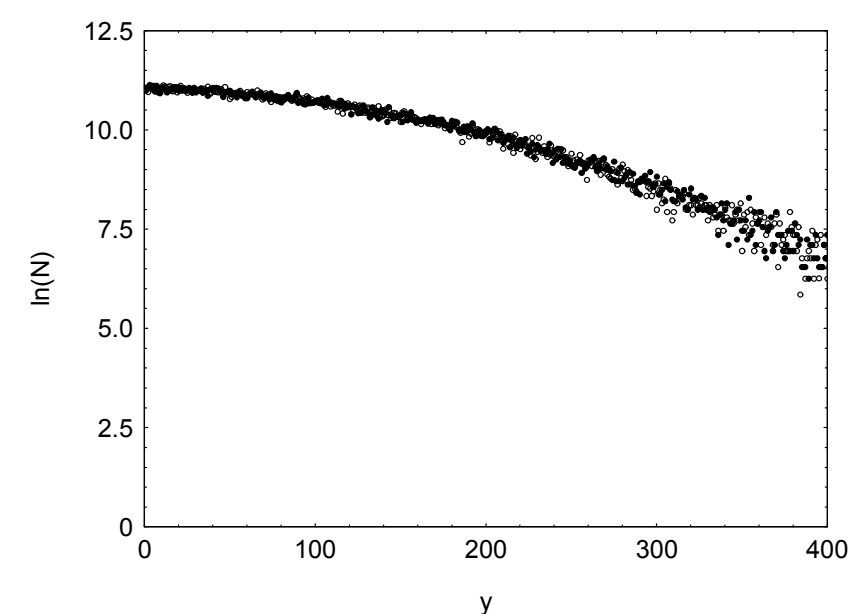

(a)

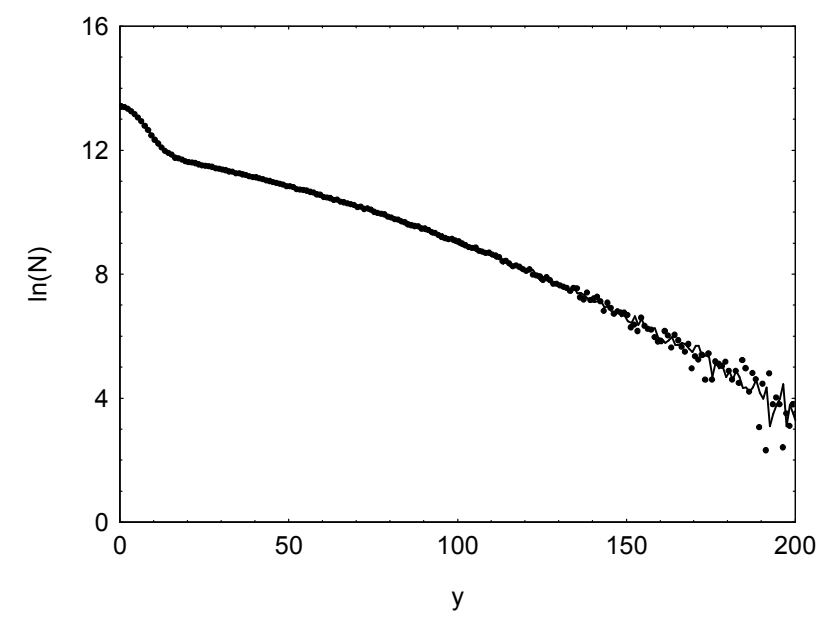

(c)

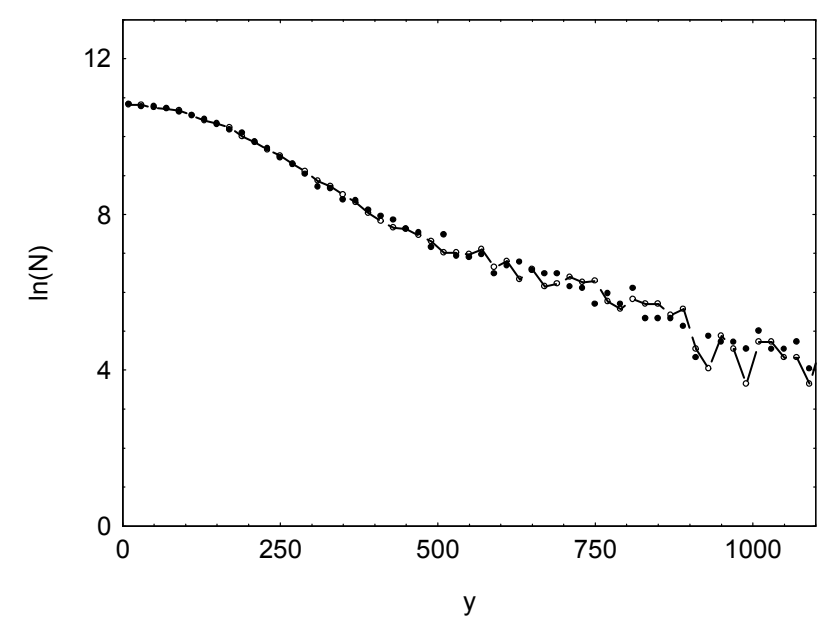

(e)

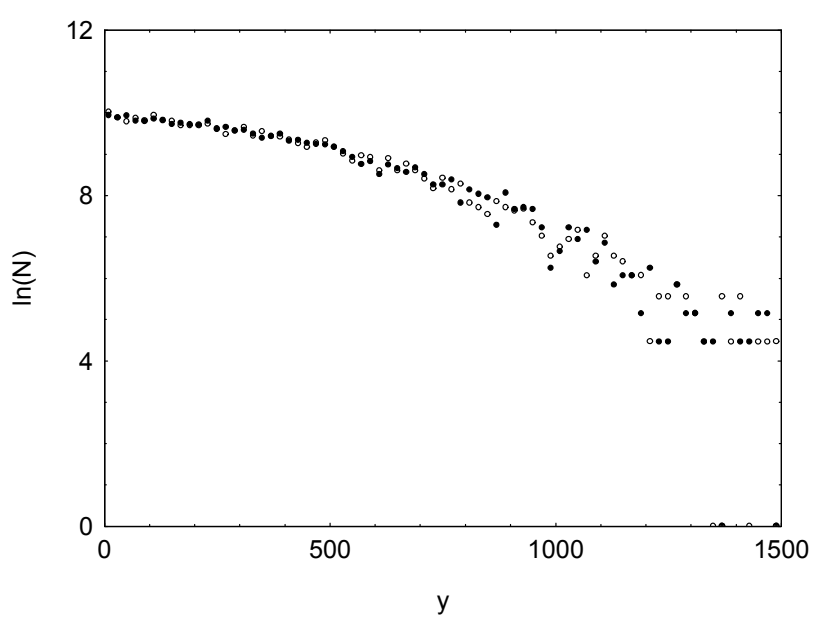

(b)

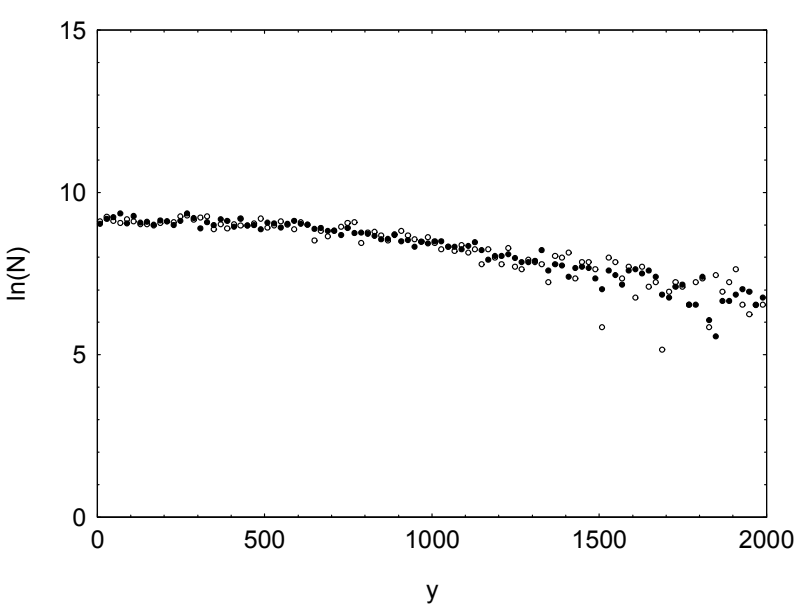

(d)

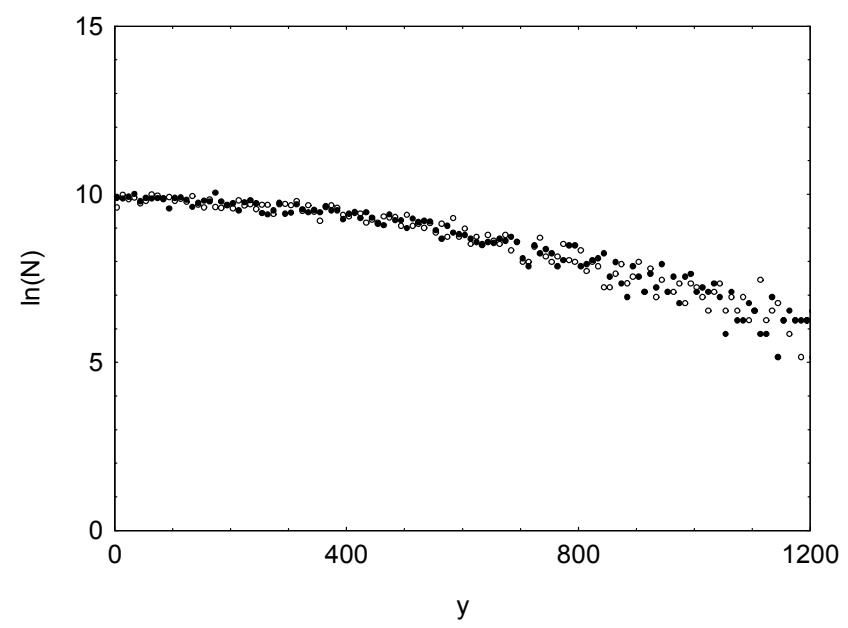

(f)

Figure 2 a-f. Concentration profiles calculated by means of the LMC method. Open symbols Model I; filled symbols - Model II. (a) C regime, $\alpha^{*}=10 ; \Lambda^{*}=22 ; \beta=10^{3} ; \mathrm{s}=10^{2}$; (b) $\mathrm{C}$ regime, $\alpha^{*}=10 ; \Lambda^{*}=22 ; \beta=10^{3} ; \mathrm{s}=10^{2}$; (c) BC regime, $\alpha^{*}=1.25 ; \Lambda^{*}=7 ; \beta=125 ; \mathrm{s}=5 ;$ (d) ABC regime, $\alpha^{*}=1.0 ; \Lambda^{*}=2.2 ; \beta=10^{2} ; \mathrm{s}=10^{2}$; (e) B regime, $\alpha^{*}=0.05 ; \Lambda^{*}=10 ; \beta=5 ; \mathrm{s}=5$; (f) A regime, $\alpha^{*}=0.1 ; \Lambda^{*}=0.56 ; \beta=10 ; \mathrm{s}=5$. 
First, we are concerned with the numerical simulations of Model I and Model II for different kinetic regimes. We used the maximum ratio of the diffusivities $\Delta=D_{\mathrm{gb}} / D_{\ell}=w_{\mathrm{gb}} / w_{1}=10^{3}$. The resulting profiles are presented in Fig 2 a-f, where open symbols are used for Model II and filled symbols are used for Model I concentration profiles.

In Fig. $3 \mathrm{a}-\mathrm{b}$ we show contributions to the total concentration profiles from the separate grains concentration profiles and grain boundaries concentration profiles. We can see that indeed the simulations confirmed that Model I and Model II produce almost identical concentration profiles.

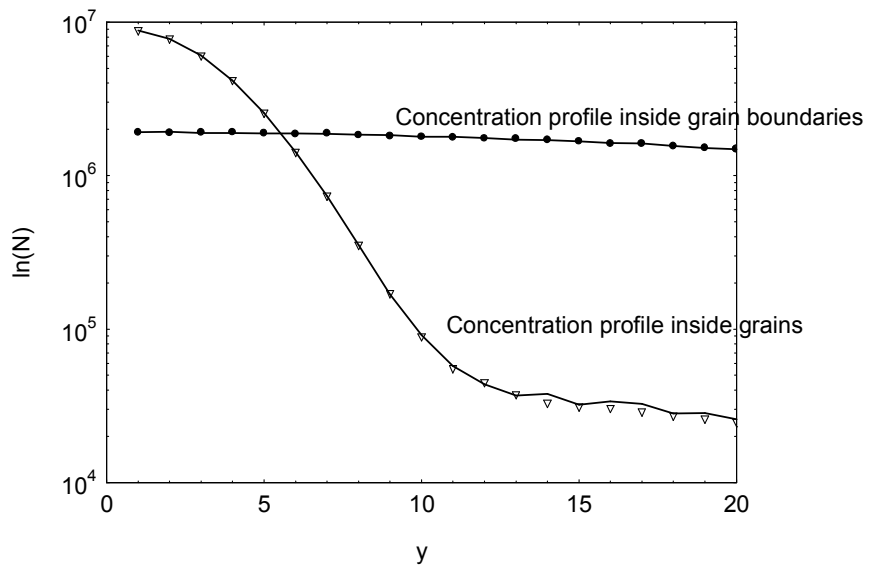

(a)

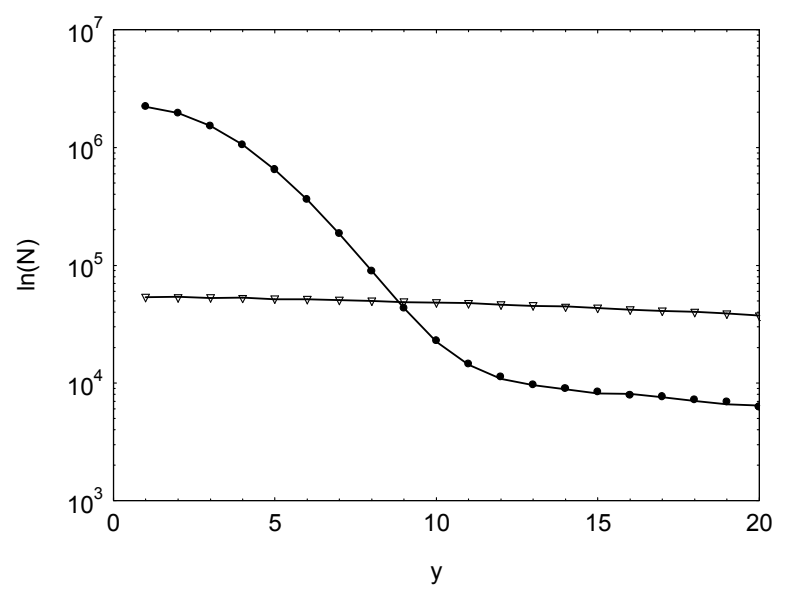

(b)

Figure 3. Contributions to the concentration depth profile from inside of the grains and from the inside of the grain boundaries separately. Symbols - Model II; solid lines - Model I. (a) segregation factor $\mathrm{s}=10^{2} ;$ (b) $\mathrm{s}=10$.

In the Type-B kinetics regime, the tail part of the concentration depth profiles from a thin-film source can be analyzed using the Suzuoka [17] or Whipple-Le Claire equations [18, 19] derived for the parallel slab model. A general expression for these solutions can be given as:

$$
P=g \frac{D_{l}^{r}}{t^{q}}\left(-\frac{\partial \ln c}{d y^{1.2}}\right)^{-w}
$$

where specific values of the parameters $g, r, q$ and $\mathrm{w}$ for these equations are given in [8]. For the Suzuoka solution, when $\beta<10^{4}$, these parameters are: $g=1.206, r=0.4916 ; q=0.5084, w=$ 1.6807 and when $\beta>10^{4}$, are: $g=1.308, r=0.5 ; q=0.5, w=1.6667$. Obviously, to use these equations, the tail region of the concentration depth profile must be analyzed using the form $\ln \mathrm{c}$ vs $y^{1.2}$.

For the evaluation of the transition point between the Harrison Type-B and the intermediate Type-BC kinetics regimes we processed the tail region of the concentration depth profiles using a fitting of $y^{1.2}$ and the Suzuoka equation. This should provide the triple product $P$ if Type-B kinetics are followed. Our strategy is to process systematically all the LMC concentration profiles obtained and plot the resulting ratio of the actual values of $P$ (used as input in the simulations) and $P_{\text {calc }}^{S}$ - the value calculated using the Suzuoka solution. The critical transition point between these two regimes was then accepted as the point where $P_{\text {calc }}^{S}$ starts to deviate from the input values of $P$.

It was found that with a substantial segregation effect (about 100 and higher) the Type-B regime is difficult to capture by means of computer simulation because of the 'shift and expansion' of the Type- $\mathrm{C}$ and $\mathrm{BC}$ regimes towards the Type-A regime. This can be demonstrated with the following set of equations. First, in computer simulations we can only deal with grain sizes of up to $50 \mu \mathrm{m}$, i.e. $d_{\max }=10^{5} \delta$. In general, computations have been made for $d_{\mathrm{av}}=0.5 \mu \mathrm{m}=10^{3} \delta$. Then we have that for the Type-B regime to exist in the average calculations we need to fulfil the following strict conditions: 


$$
\frac{d_{a v}}{3}=\frac{10^{3} \delta}{3}>\sqrt{D_{\ell} t}>\frac{10 \delta s}{2}
$$

or

$$
10^{2}>s
$$

Eq. 22 shows that a large segregation factor can significantly reduce the interval of existence for the Type-B kinetics regime. In cases of very strong segregation or relatively fine grains the Type-B kinetics regime can disappear altogether.

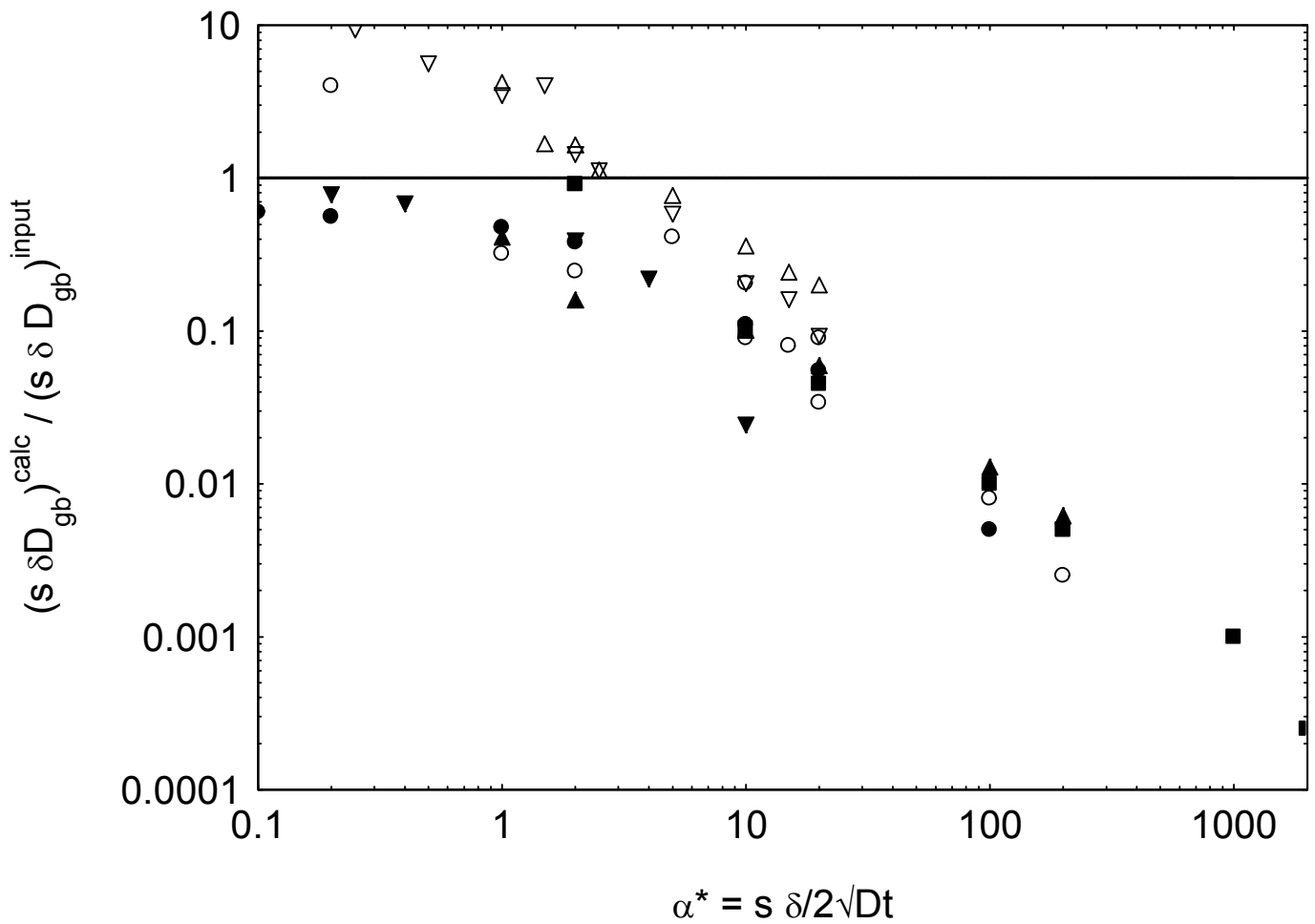

Figure 4. Ratio of the triple product as calculated using the Suzuoka solution and analyzing LMC concentration depth profiles to the input triple product as function of $\alpha^{*} . \Delta: \bullet-\Delta=10^{3} ; \mathbf{\square}-\Delta=$ $10^{2} ; \nabla-\Delta=10^{3} ; \nabla-\Delta=10^{3} ; \circ-\Delta=10^{2} ; \Delta-\Delta=10^{2} . \alpha^{*}=0.1$ is the final point for the Type-B regime. $\circ, \Delta$ and $\nabla$ - cases where the Type-B regime disappeared due to the relatively small grain size $10-100 \mathrm{~nm}$.

On the other hand, we found that the exponent of $6 / 5$ can be reached quickly enough but the resulting triple product is usually lower then expected in the $\mathrm{BC}$ intermediate regime and higher than expected in the $\mathrm{AB}$ intermediate regime, see Fig 4. These findings are consistent with the analytical derivations published in [20].

A similar strategy was used for the transition between the intermediate Type-BC and Type$\mathrm{C}$ regimes. Here we again processed the concentration depth profiles using a fitting of $y^{2}$ to obtain an estimate for the grain boundary diffusivity $D_{\mathrm{gb}}^{\mathrm{MC}}$. This should provide the input $D_{\mathrm{gb}}$ if Type-C kinetics is followed. In Figure 5 we present results of the LMC simulations for this analysis. The end of the Type-B kinetics regime and the onset of the Type-C kinetics regime were given in terms of the diffusion parameter $\alpha^{*}=s \delta / 2 \sqrt{D_{\ell} t}$. There, the critical value of $\alpha^{*}$ for the start of the Type$\mathrm{C}$ kinetics is $\alpha^{*}=5$. 


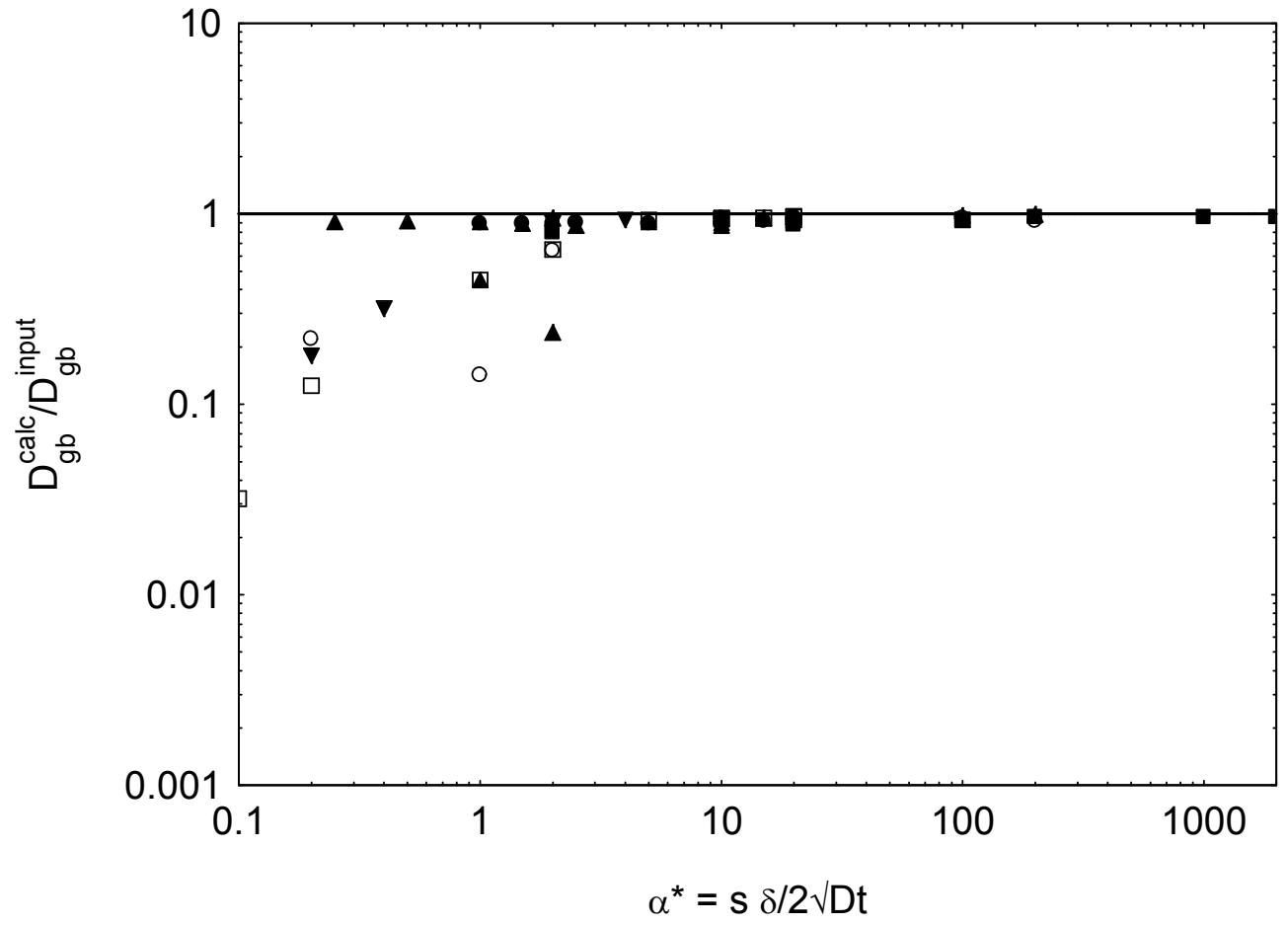

Figure 5. Ratio of the grain boundary diffusion coefficient as calculated using a Gaussian analysis of the LMC concentration depth profiles to the input triple product as function of $\alpha^{*} . \Delta: \bullet-\Delta=$ $10^{3} ; \boldsymbol{\square}-\Delta=10^{2} ; \nabla-\Delta=10^{3} ; \nabla-\Delta=10^{3} ; \circ-\Delta=10^{2} ; \Delta-\Delta=10^{2} . \alpha^{*}=5.0$ is the beginning point for the Type-C regime.

\section{Summary}

For the case of impurity grain boundary diffusion, segregation is very important. In the present study, the influence of segregation on the transition points for the three main Harrison kinetics regimes (A, B and $\mathrm{C}$ ) for the measurement of grain boundary diffusivities from impurity concentration depth profiles has been investigated for the parallel slab model (essentially 2dimensional) by making use of the tracer-type solution to the equivalent diffusion problem. It was shown that the Type-B regime is most likely not realized for the cases of fine-grained material with a strong segregation effect present.

\section{Appendix}

First, we point out that both Models must have solutions that are symmetric with respect to $x=0$. Therefore the Taylor series expansion for the Model I grain boundary concentration $c_{\mathrm{b}}$ should be written as follows:

$$
\begin{aligned}
c_{b}(x, y, t) & =c_{b}(0, y, t)+\left.\frac{x^{2}}{2} \frac{\partial^{2} c_{b}(x, y, t)}{\partial x^{2}}\right|_{x=0}+o\left(x^{4}\right) \\
& =c_{b 0}+\frac{x^{2}}{2} c_{b 2}+o\left(x^{4}\right)
\end{aligned}
$$

Substituting (A1) into boundary conditions Eq. 11 we have that:

$$
D_{g b} c_{b 2}=\left.\frac{2 D_{\ell}}{\delta} \frac{\partial c_{g}}{\partial x}\right|_{x=\delta / 2} .
$$

Then, after substituting Eq.10, A1, A2 into Eq. 8 at $x=\delta / 2$ we have that: 


$$
\frac{\partial c_{g}}{\partial t}=\frac{2 D_{\ell}}{s \delta} \frac{\partial c_{g}}{\partial x}+\left.D_{g b} \frac{\partial^{2} c_{g}}{\partial y^{2}}\right|_{x=\delta / 2}
$$

As was noted in [8] the mathematical problem for diffusion inside the grain thus reduced to the solution of Eq. 9 with the condition A3 containing only $c_{\mathrm{g}}$.

A similar technique applied for Model II gives us exactly the same mathematical problem for $c_{\mathrm{g}}$.

Now if we look at $c_{b}$ again for Model I ( $\left.c_{b I}\right)$ and Model II ( $\left.c_{b I I}\right)$ then we will have that condition Eq. 11 and condition Eq. 16 can only be fulfilled if :

$$
c_{\mathrm{b} 2 \mathrm{I}}=s c_{\mathrm{b} 2 \mathrm{II}}
$$

On the other hand, the condition Eq.10 and condition Eq. 15 can only be fulfilled if:

$$
c_{\mathrm{b} 0 \mathrm{I}}=s c_{\mathrm{b} 0 \mathrm{II}} \quad \text { and } \quad c_{\mathrm{b} 2 \mathrm{I}}=s^{3} c_{\mathrm{b} 2 \mathrm{II}}
$$

where the second equation contradicts Eq. A4. Therefore Model I and Model II will differ only for the grain boundary concentration solution and the difference between the Models will be of the order $\delta^{2}$.

\section{Acknowledgements}

We acknowledge the Australian Research Council for its support of this work.

\section{References}

[1] L.G. Harrison, Trans. Farad. Soc. Vol. 57 (1961), p. 1191.

[2] I.V. Belova and G.E. Murch, Phil. Mag. Vol. 81 (2001), p. 2447.

[3] S.V. Divinski, F. Hisker, Y.-S. Kang, J.-S. Lee and Chr. Herzig., Z. Metallk. Vol. 93 (2002), p. 256.

[4] I.V. Belova and G.E. Murch, Defect and Diffusion Forum Vol. 258/260 (2006), p. 483.

[5] I V Belova and G E Murch, Defect and Diffusion Forum Vol. 273/276 (2008), p. 425.

[6] I V Belova and G E Murch, Defect and Diffusion Forum Vol. 283/286 (2009), p. 697.

[7] I.V. Belova and G.E. Murch, Phil. Mag. Vol. 89 (2009), p. 665.

[8] I. Kaur, Y. Mishin and W. Gust, Fundamentals of Grain and Interphase Boundary Diffusion (Wiley, Chichester 1995).

[9] S.V. Divinski, and L.N. Larikov, Defect and Diffusion Forum Vol. 143/147 (1997), p. 1469.

[10] E.W. Hart, Acta Metall. Vol. 5 (1957), p. 597.

[11] J.C. Fisher, J. Appl. Phys. Vol. 22 (1951), p. 74.

[12] B.S. Bokshtein, I.A. Magidson and I.L. Svetlov, Phys. Met. Metallogr. Vol. 6(6) (1958), p. 81.

[13] G.B. Gibbs, Phys. Stat. Solidi a Vol. 16 (1966), p. K27.

[14] J.C. Maxwell-Garnett, Phil. Trans. Roy. Soc. London, Vol. 203 (1904), p. 386.

[15] J.C. Maxwell, A Treatise on Elasticity and Magnetism, $3^{\text {rd }}$ Ed. Clarendon Press, 1892, p. 435.

[16] I.V. Belova, G.E. Murch, T. Fiedler and A. Öchsner, Diffusion Fundamentals, Vol. 4 (2007), p.15.1 (electronically published).

[17] T. Suzuoka, J. Phys. Soc. Japan, Vol. 19 (1964), p. 839.

[18] R.T.P. Whipple, Phil. Mag., Vol. 45 (1954), p. 1225.

[19] A.D. Le Claire, Brit. J. Appl. Phys. Vol. 14 (1963), p. 351.

[20] I.A. Szabo, D.L. Beke and F.J. Kedves, Phil. Mag. Vol. 62 (1990), p. 227. 


\section{Grain Boundary Diffusion, Stresses and Segregation}

10.4028/www.scientific.net/DDF.309-310

The Harrison Diffusion Kinetics Regimes in Grain Boundary Diffusion: Lattice Monte Carlo Modelling of the Effect of Segregation

10.4028/www.scientific.net/DDF.309-310.9

\section{DOI References}

[4] I.V. Belova and G.E. Murch, Defect and Diffusion Forum Vol. 258/260 (2006), p. 483.

doi:10.4028/www.scientific.net/DDF.258-260.483

[5] I V Belova and G E Murch, Defect and Diffusion Forum Vol. 273/276 (2008), p. 425.

doi:10.4028/www.scientific.net/DDF.273-276.445

[6] I V Belova and G E Murch, Defect and Diffusion Forum Vol. 283/286 (2009), p. 697.

doi:10.4028/www.scientific.net/DDF.283-286.697

[9] S.V. Divinski, and L.N. Larikov, Defect and Diffusion Forum Vol. 143/147 (1997), p. 1469.

doi:10.4028/www.scientific.net/DDF.143-147.1469

[10] E.W. Hart, Acta Metall. Vol. 5 (1957), p. 597.

doi:10.1016/0001-6160(57)90127-X

[13] G.B. Gibbs, Phys. Stat. Solidi a Vol. 16 (1966), p. K27.

doi:10.1016/S0007-0785(66)80010-8

[20] I.A. Szabo, D.L. Beke and F.J. Kedves, Phil. Mag. Vol. 62 (1990), p. 227.

doi:10.4028/www.scientific.net/DDF.66-69.427 